\title{
Technetium-99m ethylene cysteine dimer posterior fossa perfusion and brain death
}

This article was published in the following Dove Press journal:

Research and Reports in Nuclear Medicine

II September 2012

Number of times this article has been viewed

\section{Usha A Joseph' \\ Kunal Kothari ${ }^{2}$ \\ Dayna Skolkin ${ }^{3}$ \\ David Q Wan' \\ Isis W Gayed'}

'Department of Radiology and Nuclear Medicine, University of Texas Medical School at Houston, TX; ${ }^{2}$ Department of Diagnostic Radiology, University of Maryland Medical Center, Baltimore, MD; ${ }^{3}$ Bachelor of Nursing Student, University of Texas at Austin, TX, USA
Correspondence: Usha A Joseph Department of Radiology/Nuclear Medicine, The University of Texas Medical School at Houston 643। Fannin, Suite 2 130b, Houston, TX 77030, USA

Tel +I 7|35664562

Fax + I 713566428 I

Email usha.a.joseph@uth.tmc.edu
Background: The purpose of this study was to evaluate the frequency of and outcome in patients with isolated posterior fossa uptake on cerebral perfusion scan.

Methods: A retrospective review was undertaken of electronic medical records over 6 years from 713 consecutive patients undergoing technetium-99m ethyl cysteine dimer cerebral perfusion scans to identify isolated posterior fossa activity.

Results: Of the 13 patients with isolated posterior fossa activity, 10 deteriorated to death and three had life support withdrawn. They survived an average of 0.24 day \pm 0.27 standard deviation (SD) days after scanning and an average of $1.03 \pm 0.33 \mathrm{SD}$ days on life support.

Conclusion: Unilateral or bilateral isolated posterior fossa technetium-99m ethyl cysteine dimer activity indicates imminent or impending brain death and a poor prognosis.

Keywords: isolated posterior fossa activity, cerebral perfusion scan, brain death, technetium- $99 \mathrm{~m}$ ethyl cysteine dimer

\section{Introduction}

Brain death is determined on the basis of a comprehensive clinical examination by a skilled physician and is defined as the irreversible loss of function of the entire brain including the brain stem resulting from irreversible cessation of circulation and respiration or cessation of function of the entire brain and brainstem. ${ }^{1}$ Ancillary confirmatory tests such as electroencephalograms, measurement of somatosensory evoked potentials, and cerebral blood flow evaluation by contrast angiography, transcranial Doppler ultrasound, or radionuclide cerebral angiography are optional studies performed to supplement an incomplete physical examination or if a reliable clinical examination cannot be performed. ${ }^{1}$

Radionuclide cerebral angiography using hydrophilic agents has been used to evaluate brain death since the late 1960s but recently hydrophilic agents have been mostly replaced by lipophilic agents, ${ }^{2}$ with the absence of cerebral as well as cerebellar flow and perfusion consistent with the clinical diagnosis of brain death. Contradictory findings of preserved infratentorial/cerebellar perfusion with absent cerebral (supratentorial) perfusion are rare. Valle et $\mathrm{al}^{3}$ called such findings "a step in the brain death phenomenon" and felt that brain death could not be confirmed under these circumstances. ${ }^{3}$ Others have found that death results within hours to days after the findings in such patients. ${ }^{2}$ Radionuclide angiography is a sensitive easily performed test to confirm the clinical diagnosis of brain death but may not adequately evaluate the posterior fossa circulation; an occasional patient may show residual brain stem function on brain scan. ${ }^{4}$ 
However, it is a reliable test in deeply comatose patients with no brain stem reflex and patients for whom formal apnea tests are inconclusive. ${ }^{5}$ Withdrawal of sedative drugs and arbitrary waiting periods are not required for performance of radionuclide angiography. ${ }^{6}$

Technetium-99m (Tc-99m) ethyl cysteine dimer and technetium-99m hexamethyl propylene amine oxime (HMPAO) are nondiffusible lipophilic brain imaging agents with quantitatively similar cerebral kinetics, initial distribution, and rapid uptake in the gray matter (although HMPAO is retained longer than ethyl cysteine dimer). Cerebral flow and uptake in the parenchymal gray matter of these agents is proportional to perfusion, and the agents stay trapped within the gray matter cells for some time. ${ }^{2}$ We use Tc-99m ethyl cysteine dimer for brain imaging because the brain-to-soft tissue activity ratios continue to increase over time resulting in less extracerebral activity, making it easier to interpret ethyl cysteine dimer images than HMPAO images. ${ }^{7}$

We found isolated posterior fossa activity on a Tc-99m ethyl cysteine dimer cerebral perfusion scan in one patient and so undertook a retrospective chart review of consecutive cerebral perfusion scans performed at our level one trauma hospital over a 6-year period to determine the frequency and patient outcome of isolated posterior fossa/infratentorial activity seen on Tc-99m ethyl cysteine dimer cerebral perfusion scans.

\section{Materials and methods}

After obtaining approval from the institutional review boards of the hospital and medical school, relevant electronic medical records and cerebral perfusion scan reports in all 713 consecutive patients undergoing one or more cerebral perfusion scans from January 2003 through February 2009 were retrospectively accessed and analyzed.

The images of patients with isolated posterior fossa activity were reviewed by the lead author. Concurrence of scan findings with the reported findings was confirmed in all the patients. The patients with isolated posterior fossa activity were analyzed regarding age, gender, type of brain injury, days on life support, and days survived after the nuclear cerebral perfusion scan. Each adult patient had been intravenously injected with 20-28 mCi (740-1036 MBq) of Tc-99m ethyl cysteine dimer (in pediatric patients, the dose was based on patient weight in kilograms). Quality control measures for each dose of radiopharmaceutical were performed before patient administration. After intravenous bolus injection of the radiotracer, anterior dynamic flow images of the head were acquired for 90 seconds at one second per frame, starting when activity was seen in the carotid arteries in the neck using a portable Adac TransCam gamma camera with a low energy diverging $12^{\prime \prime}$ diameter collimator at the patient's bedside. The flow imaging was followed by anterior blood pool imaging and 20-minute delayed anterior and lateral planar spot images of the head acquired for 3 minutes per image. The images were initially viewed on X-ray films and later on PACS monitors. Data were collected from patient electronic medical records, and the images of the 13 patients with posterior fossa activity were reviewed by a nuclear medicine physician.

\section{Results}

Review of the reports of cerebral perfusion findings in 713 consecutive patients showed that in the majority of patients $(567 / 713,79.5 \%)$, the initial brain scan findings confirmed the clinical diagnosis of brain death. One hundred and twenty-eight of 713 patients showed variable cerebral uptake on the initial scan, and in 45 of these 128 patients, a repeat brain scan showed findings consistent with brain death. Of the 713 patients, 13 patients (1.82\%) had seemingly contradictory isolated posterior fossa activity on cerebral perfusion imaging (arrows in Figure 1) and no intracerebral flow or perfusion of Tc-99m ethyl cysteine dimer (ie, a "mismatched finding").

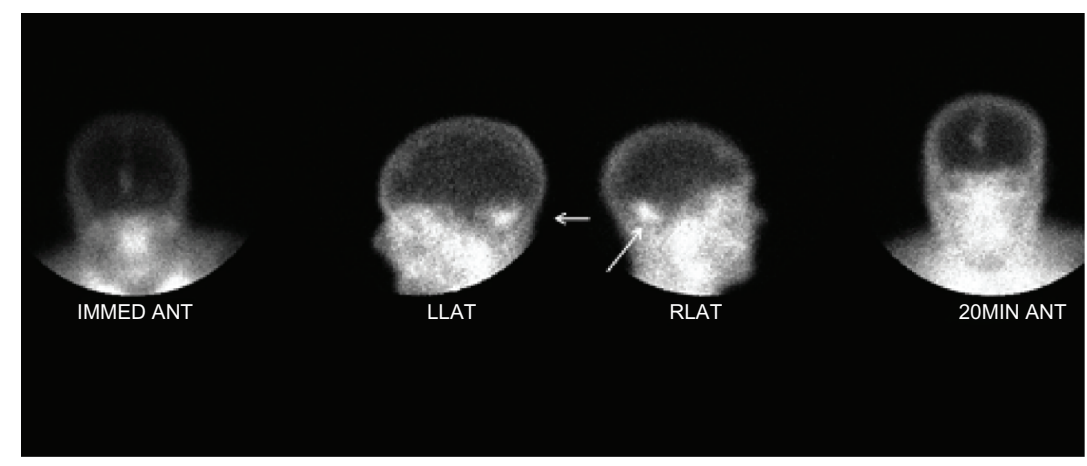

Figure I A 66-year-old male comatose after a fall.

Notes: Cerebral perfusion scan after $27 \mathrm{mCi}(999 \mathrm{MBq})$ of technetium-99m ethyl cysteine dimer intravenously showing focally increased uptake in the bilateral posterior fossa, best seen on the lateral projections (arrows). No activity is seen in the bilateral cerebral hemisphere on the immediate and 20-minute delayed images. The small focus of uptake in the frontal region near the midline is most likely superficial scalp activity. 
The 13 patients were 11 males and two females. The males were aged $12-87$ years and the women were aged 44-57 years. The mean age of all 13 patients was 45.85 years \pm 22.45 standard deviation (SD). The average number of days on life support was 1.03 days $\pm 0.33 \mathrm{SD}$. The medical condition of all 13 patients deteriorated after the scans. Ten patients eventually died from cardiocirculatory arrest and nonsalvageable massive neurologic insult. Life support was withdrawn from the other three patients at the request of their families after comprehensive clinical examinations showed brain death.

The 13 patients lived an average of 0.24 days \pm 0.27 $\mathrm{SD}$ after their cerebral perfusion scans, which was approximately $23 \%$ of their total time on life support. Ten of the 13 comatose patients had suffered traumatic brain injuries from motor vehicle accidents, gunshot wounds to the head, or falls resulting in subdural hematoma in five patients, intracranial hemorrhage in three patients, subarachnoid hemorrhage and subdural hematoma in one patient, and diffuse cerebral edema and massive brain injury from gunshot to the head in one patient; many showed a combination of brain insults like skull fractures, cerebral edema, and intracranial hemorrhage, and in three patients coma resulted from medical conditions such as cerebrovascular accident (Table 1).

Figure 2 shows intense physiologic parotid gland activity (short arrow) below and separate from posterior fossa activity (long arrow). The parotid activity may be difficult to separate from posterior fossa activity in other patients (Figure 3A, arrow).

One patient had a repeat brain scan 8 hours after the first scan at the request of the patient's neurologist because the patient, a potential cadaver organ donor, was deemed brain dead by clinical evaluation, and waiting the recommended time of 24 hours for repeat imaging would compromise the integrity of any organs harvested. Repeat imaging in this patient (Figure 3B) revealed persistent but milder posterior fossa activity on the anterior and left lateral projection that probably represents persistent residual posterior fossa activity, which is less intense when compared with the parotid gland activity (long arrow in Figure 3B). Anterior and left lateral static images obtained to check for residual background activity before injecting Tc-99m ethyl cysteine dimer, revealed mild focal activity in the posterior fossa region on the left lateral image (thick arrow in Figure 3B). Intense parotid activity is again seen on the repeat scan (short arrow in Figure 3B).

\section{Discussion}

Radionuclide cerebral perfusion imaging is a simple and useful ancillary test for confirming the clinical diagnosis of brain death. It is convenient, available at all times (including week nights and weekends) in most major hospitals, and is easily performed at the patient's bedside with a portable gamma camera. In our series of 713 patients seen over a 6-year period, 13 patients (1.8\%) had isolated posterior fossa activity on Tc-99m ethyl cysteine dimer cerebral perfusion scans. These 13 patients were on life support for an average of 1.03 days \pm 0.33 SD days and survived for an average 0.24 day $\pm 0.27 \mathrm{SD}$ after their scans. The medical conditions of all 13 patients continued to deteriorate, and eventually 10 died from cardiocirculatory arrest and nonsalvageable massive neurologic insult; life support was removed from the other three patients at the request of their families after a comprehensive clinical examination showed brain death. One patient had a repeat brain scan 8 hours after the first scan that showed persistent but milder activity in the posterior fossa that probably represented residual decreasing posterior fossa activity from the earlier study. A prior report showed that death resulted within hours to days later in such patients. ${ }^{2}$

Determination of brain death is made on the basis of comprehensive clinical assessment by a skilled physician, but confounding factors in some patients can make the clinical examination unreliable, necessitating additional ancillary testing for confirmation of brain death. Most hospitals have the capability to perform and accurately interpret ancillary tests, such as electroencephalography, radionuclide brain flow/perfusion scanning, and X-ray contrast angiography. Contrast angiography is accurate but invasive, and single

Table I Characteristics of 13 patients with isolated posterior fossa technetium-99m ethyl cysteine dimer activity

\begin{tabular}{|c|c|c|c|c|c|c|}
\hline Gender & Age, years & Coma & $\begin{array}{l}\text { Mean }( \pm S D) \text { days } \\
\text { on ventilator }\end{array}$ & $\begin{array}{l}\text { Mean }( \pm S D) \text { days } \\
\text { survived beyond scan }\end{array}$ & Organ donor & $\begin{array}{l}\text { Outcome in } \\
13 \text { patients }\end{array}$ \\
\hline $\begin{array}{l}\text { II males } \\
2 \text { females }\end{array}$ & $\begin{array}{l}\text { Males, } \\
\text { I2-87 years } \\
\text { Females, } \\
44-57 \text { years }\end{array}$ & $\begin{array}{l}\text { *Traumatic brain injury } \\
(n=10) \\
\text { Cerebrovascular accident } \\
(n=3)\end{array}$ & $\mathrm{I} .03$ days \pm 0.33 & 0.24 day \pm 0.27 & $8 / 13$ patients & $\begin{array}{l}\text { 10/13 expired from } \\
\text { cardiocirculatory arrest } \\
\text { and nonsalvageable } \\
\text { neurologic insult } \\
\text { 3/13: life support } \\
\text { withdrawn }\end{array}$ \\
\hline
\end{tabular}

Note: *Subdural hematoma, intracranial hemorrhage, subarachnoid hemorrhage, skull fractures, cerebral edema, and combination of brain injuries in some patients. Abbreviation: SD, standard deviation. 


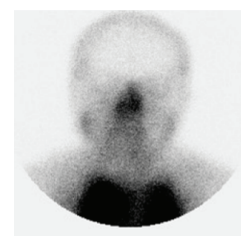

IMMED ANTERIOR

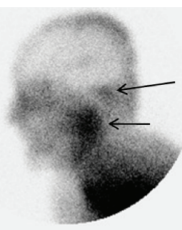

LLAT

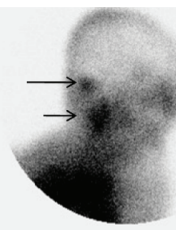

RLAT

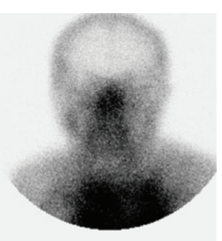

20MIN ANTERIOR

Figure 2 A 57-year-old female comatose after head trauma

Notes: Immediate and 20-minute delayed planar images of the head after $23.8 \mathrm{mCi}(880.6 \mathrm{MBq})$ of intravenous technetium-99m ethyl cysteine dimer showing focally increased activity in the posterior fossa, right worse than left (long arrow). No activity is seen in the bilateral cerebral hemisphere. Focal intense physiologic uptake in the bilateral parotid glands is seen below and separate from the posterior fossa activity (small arrow).
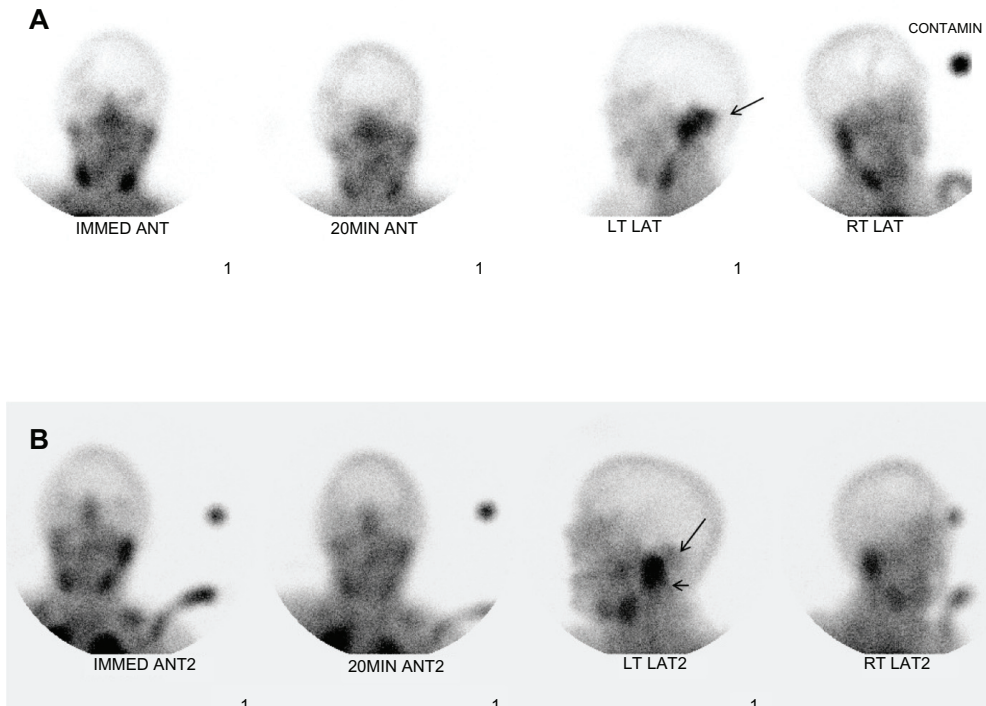

LT LAT2

8HR REPEAT STUDY

ALL IMAGES SAME INTENSITY

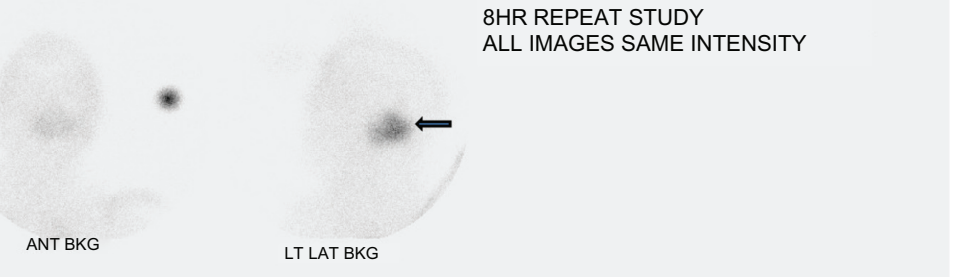

Figure 3 A 44-year-old female comatose after motor vehicle accident. (A) Immediate and 20-minute delayed planar images of the head after $28 \mathrm{mCi}$ (I036 MBq) of intravenous technetium- $99 \mathrm{~m}$ ethyl cysteine dimer showing intense focal uptake in the posterior fossa/parotid region on the left side in the left lateral projection that is probably from combined posterior fossa and physiologic parotid uptake, which are difficult to separate (arrow). (B) A technetium-99m ethyl cysteine dimer scan repeated 8 hours later showing mild focal activity in the posterior fossa in the left lateral projection (long arrow) milder in intensity than the first scan and less intense activity than parotid gland activity, short arrow, making it easier to differentiate the two.

Notes: This is probably persistent, decreased residual posterior fossa activity from the first scan, and is seen on the images obtained to evaluate residual brain background activity before the injection of technetium-99m ethyl cysteine dimer for the repeat scan (thick arrow). Intense physiologic parotid gland activity (short arrow) is again seen anterior and below the posterior fossa activity. 
photon emission computed tomography imaging although better at visualizing infratentorial activity cannot be performed at the patient's bedside. The diagnosis of brain death must be certain, because it may trigger irrevocable steps of discontinuing life support and initiating organ harvesting in those scheduled for organ donation. ${ }^{8}$ Supratentorial and infratentorial regions should be systematically and carefully evaluated in all brain scans for brain activity. Care should be taken not to confuse physiologic parotid gland activity with posterior fossa activity, because both can be present in the same patient. All of our patients demonstrating cerebral perfusion also demonstrated cerebellar perfusion.

\section{Conclusion}

The rare finding of unilateral or bilateral isolated posterior fossa activity on Tc-99m ethyl cysteine dimer brain scan is a step in the brain death process and indicates a very poor prognosis for the patient. In such circumstances, prolonging life support is a delicate decision and should always be based on the findings of a comprehensive clinical evaluation, taking into consideration legal, moral, ethical, and religious considerations, and be made with the full approval and consent of the patient's family.

\section{Disclosure}

The authors report no conflicts of interest in this work.

\section{References}

1. Zuckier LS, Kolana J. Radionuclide studies in the determination of brain death: criteria, concepts and controversies. Semin Nucl Med. 2008;38(4): 262-273.

2. Conrad GR, Sinha P. Scintigraphy as a confirmatory test of brain death. Semin Nucl Med. 2003;33(4):312-323.

3. Valle G, Ciritella P, Bonetti MG, et al. Considerations of brain death on a SPECT cerebral perfusion study. Clin Nucl Med. 1993;18(11): 953-954.

4. Flowers WM Jr, Patel BR. Radionuclide angiography as a confirmatory test for brain death: a review of 229 studies in 219 patients. South Med J. 1997;90(11):1091-1096.

5. Flowers WM Jr, Patel BR. Accuracy of clinical evaluation in determination of brain death. South Med J. 2000;93(2):203-206.

6. Goodman JM, Heck LL, Moore BD. Confirmation of brain death with portable isotope angiography: a review of 204 consecutive cases. Neurosurgery. 1985;16(4):492-497.

7. Léveillé J, Demonceau G, Walovitch RC. Intrasubject comparison between technetium-99-ECD and technetium-99m-HMPAO in healthy human subjects. $J$ Nucl Med. 1992;33(4):480-484.

8. Bonetti MG, Ciritella P, Valle G, Perrone E. 99mTc HM-PAO brain perfusion SPECT in brain death. Neuroradiology. 1995;37(5): $365-369$.
Research and Reports in Nuclear Medicine

\section{Publish your work in this journal}

Research and Reports in Nuclear Medicine is an international, peerreviewed, open access journal publishing original research, reports, reviews and commentaries on all areas of nuclear medicine. The manuscript management system is completely online and includes a very

\section{Dovepress}

quick and fair peer-review system. Visit http://www.dovepress.com/ testimonials.php to read real quotes from published authors. 